\title{
Anion transport as a target of adaption to perchlorate in sulfate- reducing communities
}

\author{
Magdalena K. Stoeva ${ }^{1,2}$ - Jennifer Kuehl ${ }^{3,4}$ - Alexey E. Kazakov ${ }^{3} \cdot$ Ouwei Wang ${ }^{1,2} \cdot$ Rowena Rushton-Green $^{1,5}$. \\ John D. Coates ${ }^{1,2,3}$
}

Received: 23 May 2019 / Revised: 4 September 2019 / Accepted: 12 September 2019 / Published online: 28 October 2019

(c) The Author(s), under exclusive licence to International Society for Microbial Ecology 2019

\begin{abstract}
Inhibitors can be used to control the functionality of microbial communities by targeting specific metabolisms. The targeted inhibition of dissimilatory sulfate reduction limits the generation of toxic and corrosive hydrogen sulfide across several industrial systems. Sulfate-reducing microorganisms (SRM) are specifically inhibited by sulfate analogs, such as perchlorate. Previously, we showed pure culture SRM adaptation to perchlorate stress through mutation of the sulfate adenylyltransferase, a central enzyme in the sulfate reduction pathway. Here, we explored adaptation to perchlorate across unconstrained SRM on a community scale. We followed natural and bio-augmented sulfidogenic communities through serial transfers in increasing concentrations of perchlorate. Our results demonstrated that perchlorate stress altered community structure by initially selecting for innately more resistant strains. Isolation, whole-genome sequencing, and molecular biology techniques allowed us to define subsequent genetic mechanisms of adaptation that arose across the dominant adapting SRM. Changes in the regulation of divalent anion:sodium symporter family transporters led to increased intracellular sulfate to perchlorate ratios, allowing SRM to escape the effects of competitive inhibition. Thus, in contrast to pure-culture results, SRM in communities cope with perchlorate stress via changes in anion transport and its regulation. This highlights the value of probing evolutionary questions in an ecological framework, bridging the gap between ecology, evolution, genomics, and physiology.
\end{abstract}

\section{Introduction}

Specific inhibitors, targeting a particular metabolism in a diverse community, represent a promising strategy for controlling the generation of harmful microbial metabolic

Supplementary information The online version of this article (https:// doi.org/10.1038/s41396-019-0540-7) contains supplementary material, which is available to authorized users.

John D. Coates

jdcoates@berkeley.edu

1 Plant and Microbial Biology, University of California, Berkeley, Berkeley, CA, USA

2 Energy Biosciences Institute, Berkeley, CA, USA

3 Environmental Genomics and Systems Biology Division, Lawrence Berkeley National Laboratory, Berkeley, CA, USA

4 Dept. of Bioengineering, University of California, Berkeley, Berkeley, CA, USA

5 Department of Microbiology and Immunology, University of Otago, Dunedin, New Zealand end-products [1-3]. One such harmful byproduct is the toxic and corrosive compound hydrogen sulfide $\left(\mathrm{HS}^{-}\right.$), which is primarily produced through the respiration of sulfate $\left(\mathrm{SO}_{4}{ }^{2-}\right)$ by dissimilatory sulfate-reducing microorganisms (SRM). SRM form a crucial link between the carbon and sulfur cycles, and are prevalent in anoxic environments [4]. Further, they pose significant economic, environmental, and human health consequences, particularly through corrosion of infrastructure used by the oil and gas, and municipal wastewater treatment industries [5]. Efforts to develop potent and specific inhibitors of SRM have identified strong inhibition by sulfate analogs such as molybdate, nitrate, and more recently, perchlorate [2, 6-10].

The sulfate analog perchlorate $\left(\mathrm{ClO}_{4}^{-}\right)$is a specific inhibitor of SRM which targets the central pathway for sulfate reduction, competitively inhibiting sulfate turnover by the sulfate adenylyltransferase (Sat) [7, 11-14]. Sat catalyzes the first step in sulfate reduction, using ATP to activate sulfate to adenosine phosphosulfate, which is subsequently reduced to sulfite and sulfide. In the model SRM Desulfovibrio alaskensis G20, perchlorate stress can be relieved through inactivation of the rex gene, a redox-sensitive transcriptional regulator, that 
represses sat and other genes central to sulfate respiration $[9,15,16]$. The de-repression of sat increases perchlorate resistance by providing more enzymes for sulfate turnover $[9,15,16]$. Non-intuitively, D. alaskensis G20 can also adapt to $100 \mathrm{mM}$ perchlorate via a single base pair substitution in sat $(\operatorname{sat}(709 \mathrm{~T}>\mathrm{G}))$ [17]. In vitro characterization of the wild type and mutant sat reveals that this substitution decreases perchlorate-mediated inhibition of sulfate turnover approximately threefold. While sat is a target of inhibition by perchlorate in pure culture, previous work has failed to detect adaptation of sulfate-reducing communities to this inhibitor [18]. Thus, the relevance of $\operatorname{sat}(709 \mathrm{~T}>\mathrm{G})$ in an ecological context, where the cost/benefit balance of mutations in a key respiratory gene may be shifted, remains unknown.

Adaptive laboratory evolution is an experimental approach that can identify mechanisms of adaptation across various contexts [19-23]. Recent studies highlight the importance of integrating ecology and evolution by studying adaptation in ecologically-relevant contexts; prediction of evolutionary outcomes must consider the diversity and composition of communities [24-27]. More recently, Lenski outlined the ESENCE method-'embedded species evolving in a natural community' - as an approach for expanding adaptive evolution studies to communities [28]. In the case of perchlorate, understanding both targets and mechanisms of resistance in a community context is of practical value, as perchlorate is an emerging treatment for sulfide production in oil reservoirs [9, 11, 29]. Moreover, understanding targets of adaptation to perchlorate stress in new organisms provides basic insights into dissimilatory sulfate reduction, its regulation, and control over its specificity.

Here, we use adaptive evolution to probe the evolutionary trajectories of both model and non-model SRM in communities and under increasing perchlorate stress. We demonstrate the importance of ecological sorting as an initial response. Isolation, whole genome and metagenomic sequencing, and classical molecular biology techniques allow us to further identify two anion transporters critical to the genetic mechanism of adaptation in the dominant adapting SRM. In contrast to previous pure culture results, which emphasize sat as a primary target of perchlorate, we find that in a community setting, adaptation targeted anion transport and its regulation. This work emphasizes the importance of anion transport in response to sulfate analog stress in environmental systems.

\section{Methods}

\section{Sequence data availability}

All sequencing data are publicly available under the NCBI BioProject ID PRJNA560652.

\section{Adaptive evolution in communities}

Adaptive evolution experiments were performed using a sulfate-reducing community enriched from San Francisco Bay sediment in modified artificial pore water medium (APM) with $20 \mathrm{mM}$ sulfate and $2 \mathrm{~g} / \mathrm{L}$ yeast extract [18]. Cultures (3 replicates) were transferred into modified APM with $60 \mathrm{mM}$ lactate, $20 \mathrm{mM}$ sulfate, and $10 \mathrm{mM}$ perchlorate and growth of SRM was monitored by quantifying sulfide production using the Cline assay [30]. Upon reaching early log phase ( $\sim 3-5$ days), cultures were transferred at a $10 \%$ inoculum to sequentially higher concentrations of perchlorate $(5-10 \mathrm{mM}$ increments) up to $100 \mathrm{mM}$. Control cultures were transferred in parallel in the absence of perchlorate. A second set of community adaptations was augmented with $D$. alaskensis $\mathrm{G} 20$. At each transfer, $1 \mathrm{~mL}$ was pelleted $(5 \mathrm{~min}$ at $13,000 \times g)$ and immediately stored at $-20^{\circ} \mathrm{C}$ for DNA extraction, and another $1 \mathrm{~mL}$ was archived in $25 \%$ glycerol, at $-80^{\circ} \mathrm{C}$. Archived communities were recovered in the absence of perchlorate, and their resistance to the inhibitor was quantified using doseresponse curves $[9,10]$ (Supplemental Methods). DNA was extracted using a PowerBiofilm DNA Isolation Kit (Qiagen) modified to exclude bead beating, and used for 16s rRNA community profiling, and amplicon sequencing of the sat gene (Supplemental Methods).

\section{Isolation, whole genome sequencing, and variant calling}

Adapted and/or control isolates of Desulfivibrio bizertensis MKS and Desulfovibrio alaskensis G20 were obtained via serial dilutions of $100 \mathrm{mM}$ perchlorate-adapted or control communities in $1.5 \%$ agar APM plugs [31], or by plating communities on solid MOLS4 medium [32, 33]. Singlecolony isolates were purity-checked via Sanger sequencing of the 16S rRNA gene. All isolates were grown in $100 \mathrm{mM}$ perchlorate-amended medium, prior to collecting $1 \mathrm{~mL}$ of culture for DNA extraction. Due to the low abundance of $D$. bizertensis MKS in the final control communities, singlecolony isolates could not be obtained, so community DNA was used for shotgun metagenomics. DNA was extracted as previously described and submitted to the QB3 Functional Genomics Lab and Vincent J. Coates Genomics Sequencing Lab (Berkeley, CA) for library preparation and 100PE sequencing (genomic DNA) or 150PE sequencing (metagenomic DNA) on an Illumina HiSeq4000 lane. Sequencing data were used for variant calling, as previously described [17] (Supplemental Methods).

\section{RT-qPCR and RNAseq}

All primer sets used for RT-qPCR are listed in Table S1. RNA extraction, cDNA synthesis, and RT-qPCR conditions 
are described in the Supplemental Methods. RNAseq libraries of the Dde_0320::TN5 vs. wild-type G20 under no stress conditions were constructed in house, using a modified method from [34] (Supplemental Methods). A second round of RNAseq was performed under "stress" conditions (100 mM perchlorate), by the Functional Genomics Lab (QB3, Berkeley). Analysis was done using RockHopper [35].

\section{Pure-culture experiments}

Adaptation in the community context was compared with adaptation in pure culture. To this end, a pure-culture laboratory evolution experiment was performed (as described above) using the ancestral Desulfovibrio bizertensis strain MKS (isolated as described above, prior to any adaptive evolution experiments): five adapting populations were compared with two control populations. For pure culture experiments, growth was monitored via optical density at $600 \mathrm{~nm}$ (Supplemental Methods).

Results from a previous pure-culture adaptation of G20 to perchlorate revealed the dominance of a mutation in the sulfate adenylyltransferase $(\operatorname{sat}(709 \mathrm{~T}>\mathrm{G}))$. We compared the relative fitness of the $\operatorname{sat}(709 \mathrm{~T}>\mathrm{G})$ mutation and the Dde_0320 null mutation in different media. Representative strains for each mutation were also directly competed in APM, with $100 \mathrm{mM}$ perchlorate, both in the presence and in the absence of the fermentative fraction of the community, and the abundance of the Dde_0320::TN mutant was tracked through successive transfers via qPCR (Supplemental Methods).

\section{Construction of overexpression strains}

D. bizertensis MKS strains overexpressing $\mathrm{TF}_{\mathrm{Pr}}$

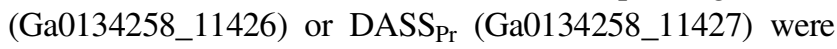
constructed as previously described [36, 37]. Complementation of sulfate uptake (via G20 genes Dde_0323-Dde_0328) was done in the E. coli CysA knock-out strain JW2415 [38]. See Supplemental Methods for respective protocols. For a full list of constructs and strains, see Table 1.

\section{Intracellular sulfate and perchlorate measurements}

Intracellular perchlorate and/or sulfate were compared between $D$. bizertensis $\mathrm{MKS}$ strains overexpressing the $\mathrm{TF}_{\mathrm{Pr}}$ transporter vs. control (P24EV and P24.1), and between wild-type G20 and the Dde_0320::TN5 mutant. In all cases, triplicate cultures ( $1 \mathrm{~L}$ for MKS and $100 \mathrm{~mL}$ for G20) of each strain were grown to $\mathrm{OD}=0.6$, pelleted and resuspended in $100 \mathrm{mM}$ perchlorate-amended APM $(10 \mathrm{~mL}$, MKS strains) or MOLS4 (40 mL, G20 strains). Cultures were incubated at $30^{\circ} \mathrm{C}$ for $1-2 \mathrm{~h}$, then pelleted and washed five times in PBS, before finally resuspending in $1 \mathrm{~mL}$ of DI water. OD600 was measured before lysing cells at $98^{\circ} \mathrm{C}$ for $30 \mathrm{~min}$. The soluble fraction was filtered $(0.2 \mathrm{um})$, diluted 1:5 in DI water, and submitted to the Andrew Jackson laboratory (Texas Tech University) for sulfate and perchlorate quantification (Supplemental Methods, [39]). Results were normalized to OD, for a final value in terms of concentration/OD.

\section{Results}

\section{Sulfate-reducing communities initially adapted to perchlorate through shifts in community structure}

We investigated the adaptation of sulfate-reducing communities to the sulfate analog perchlorate in terms of overall community structure and the physiology and genetics of the dominant taxa. Sulfate-reducing communities were adapted via serial transfers into increasing concentrations of the inhibitor (Fig. 1a). Parallel control communities were transferred in the absence of perchlorate. The inoculum consisted of a community enriched from estuary sediment that subsequently was either unaltered ("native") or biologically augmented with model SRM Desulfovibrio alaskensis G20 ("G20-augmented"). The mechanism of G20 adaptation to perchlorate has been characterized in pure culture, and thus bio-augmentation allowed us to probe the relevance of this known mechanism in a more ecologically relevant setting [17].

All replicate independently adapted native and G20augmented communities showed significantly increased resistance to perchlorate. The half maximal inhibitory concentrations (IC50s) for native adapted communities were between $90-180 \mathrm{mM}$ (95\% confidence interval of $77-224 \mathrm{mM})$ and $57-98 \mathrm{mM}$ (95\% CI: 48-121 mM) for the G20-augmented condition, compared with $22-34 \mathrm{mM}$ (95\% CI: $20-28 \mathrm{mM}$ ) and $20-26 \mathrm{mM}$ (95\%CI: $18-29 \mathrm{mM}$ ), for control native and augmented communities, respectively (Fig. 1b). Further, native adapted communities, unlike controls, displayed robust sulfidogenesis during the final transfer into $100 \mathrm{mM}$ perchlorate (Fig. S1A). G20augmented adapted communities showed varying degrees of sulfidogenesis in $100 \mathrm{mM}$ perchlorate: replicate one demonstrated no sulfide production, replicate two produced sulfide robustly, and in replicate three, only slight sulfide production occurred (Fig. S1B).

Community structure differed between native and augmented conditions, and with increasing perchlorate concentration. Control communities, both native and G20augmented, consisted of $\sim 85 \%$ Desulfovibrio spp. This community structure was stable over time, with the exception of G20 (in augmented communities), which was eventually outcompeted by native Desulfovibrio spp. 
Table 1 Plasmids/strains used in this study

\begin{tabular}{|c|c|c|}
\hline $\begin{array}{l}\text { Plasmid/ } \\
\text { Strain Name }\end{array}$ & Description & Source \\
\hline \multicolumn{3}{|l|}{ Plasmids } \\
\hline pBMK7 & $\begin{array}{l}\text { Parental plasmid; used as overexpression vector for MKS; } \\
\operatorname{Kan}^{R}\end{array}$ & $\begin{array}{l}\text { From Arash Komeili; } \\
{[37,62]}\end{array}$ \\
\hline pMS1 & 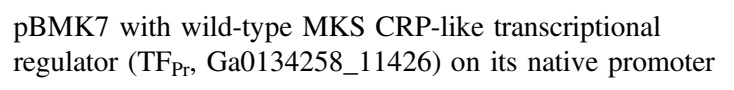 & This study \\
\hline pMS2 & $\begin{array}{l}\text { pBMK7 with the } \mathrm{P} 24 \text { mutant } \mathrm{TF}_{\mathrm{Pr}}\left(\mathrm{L} 209^{*}\right) \text { on its native } \\
\text { promoter }\end{array}$ & This study \\
\hline pMS3 & $\begin{array}{l}\text { pBMK7 with the MKS DASS } \\
\text { separate constitutive promoter (promoter for } \\
\text { Ga0134258_10740). }\end{array}$ & This study \\
\hline pOW107 & $\begin{array}{l}\text { Parental plasmid used for complementation of E. coli CysA } \\
\text { KO strain; Amp }{ }^{\mathrm{R}} \text {; weak constitutive promoter BBa_J23103 } \\
\text { (Chris Anderson, http://parts.igem.org/Promoters/Catalog/ } \\
\text { Anderson) }\end{array}$ & $\begin{array}{l}\text { From Ouwei Wang and } \\
\text { John Dueber }\end{array}$ \\
\hline pOW107.1 & pOW107 with wild-type G20 gene pclT (Dde_0326) & This study \\
\hline pOW107.2 & $\begin{array}{l}\text { pOW } 107 \text { with wildtype G20 genes of the perchlorate } \\
\text { cluster, Dde_0323 to Dde_0328 }\end{array}$ & \\
\hline \multicolumn{3}{|c|}{ Desulfovibrio bizertensis MKS strains } \\
\hline P24 & $\begin{array}{l}\text { Adapted strain of MKS with a mutation in the HTH motif of } \\
\text { the } \operatorname{TF}_{\operatorname{Pr}}\left(\mathrm{L} 209^{*}\right)\end{array}$ & This study \\
\hline wtEV & Wild type MKS with pBMK7 & This study \\
\hline wt.1 & Wild type MKS with pMS1 & This study \\
\hline wt.2 & Wild type MKS with pMS2 & This study \\
\hline P24EV & Adapted MKS strain P24 with pBMK7 & This study \\
\hline $\mathrm{P} 24.1$ & Adapted MKS strain P24 with pMS3 & This study \\
\hline \multicolumn{3}{|c|}{ Desulfovibrio alaskensis G20 strains } \\
\hline $\mathrm{G} 20$ & Wild type D. alaskensis G20 & from Adam Arkin \\
\hline Dde_0320::TN5 & G20 TN5 mutant; transposon insertion in pclS (Dde_0320) & {$[15]$} \\
\hline Dde_0321::TN5 & G20 TN5 mutant; transposon insertion in Dde_0321 & {$[15]$} \\
\hline Dde_0326::TN5 & G20 TN5 mutant; transposon insertion in pclT (Dde_0326) & {$[15]$} \\
\hline Pat3 & $\begin{array}{l}\text { G20 strain with } \operatorname{sat}(709 \mathrm{~T}>\mathrm{G}) \text { mutation; from previous pure- } \\
\text { culture adaptation }\end{array}$ & {$[17]$} \\
\hline $\operatorname{Pg} 11$ & G20 community-adapted strain with $\operatorname{sat}(709 \mathrm{~T}>\mathrm{G})$ mutation & This study (Table S4) \\
\hline Pg33 & $\begin{array}{l}\text { G20 community-adapted strain with transposon insertion in } \\
\text { pclS (Dde_0320) }\end{array}$ & This study (Table S4) \\
\hline \multicolumn{3}{|c|}{ E. coli JW2415 strains (CysA KO) } \\
\hline jwEV & JW2415 with pOW107 & This study \\
\hline jw.1 & JW2415 with pOW107.1 & This study \\
\hline jw.2 & JW2415 with pOW107.2 & This study \\
\hline
\end{tabular}

(Fig. 1c). G20-augmentation also increased Desulfovibrio bizertensis strain MKS abundance, as compared with native controls. In contrast, adapting communities started with the same structure but changed as perchlorate concentration increased. In all three native adapting communities, at 20-40 mM perchlorate a population of Desulfocurvus increased as most Desulfovibrio spp. decreased, and above $40 \mathrm{mM}$ a population of MKS outcompeted Desulfocurvus, ultimately expanding to cover the entire SRM niche. Augmented communities also saw a decrease in various native Desulfovibrio spp. and a concomitant increase in G20, which came to occupy a majority of the SRM niche. However, variability among independent replicates was greater in augmented communities: in replicate one G20 was largely displaced by a non-sulfate-reducing gammaproteobacterium Psychromonas (explaining the lack of sulfide production in the final $100 \mathrm{mM}$ transfer). In replicates two and three, G20 was sustained until the final transfer. Replicate two, where sulfidogenesis was most robust, most closely resembled native communities, showing a similar expansion of Desulfocurvus and ultimate rise of MKS. 
A.
A.
serial transfers
Isolates

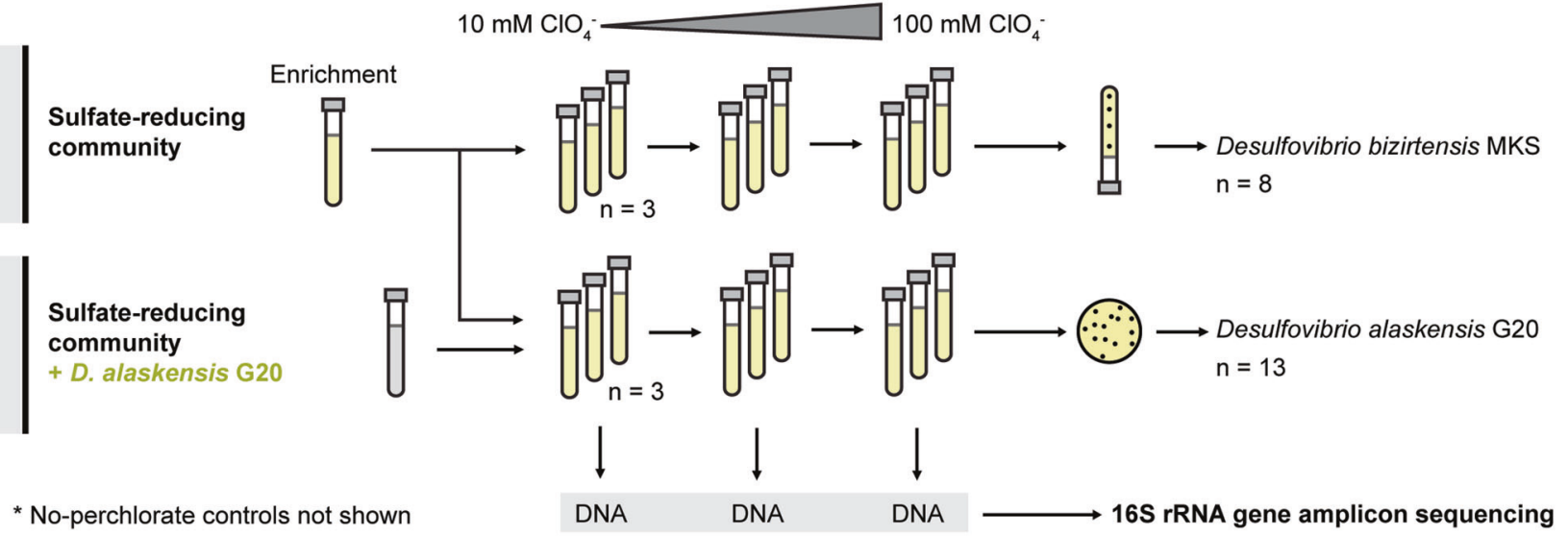

B.
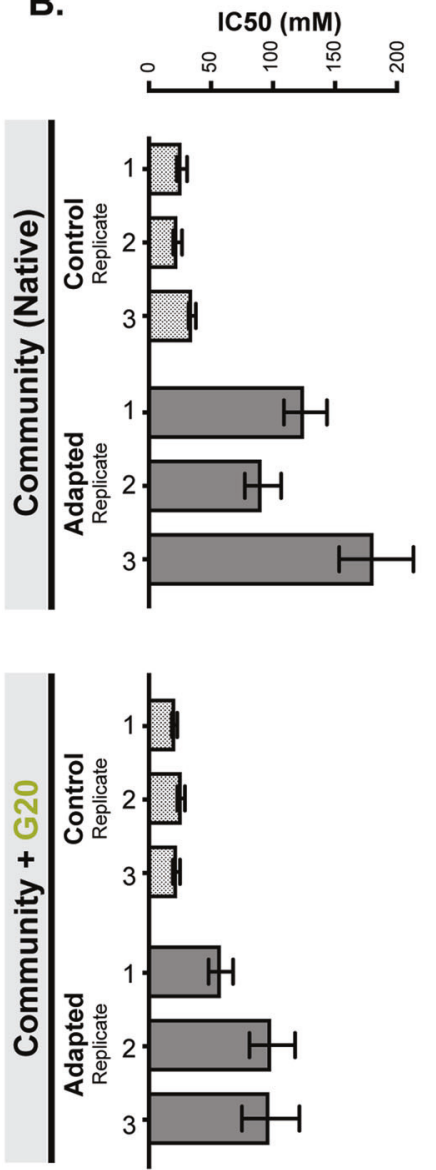

C.

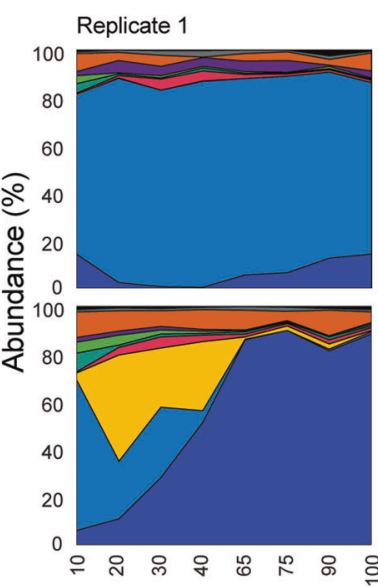

Replicate 2

Replicate 3

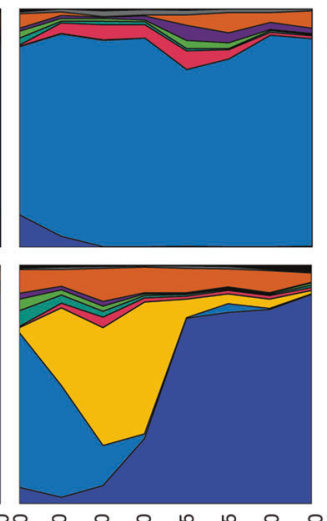

D. bizertensis MKS
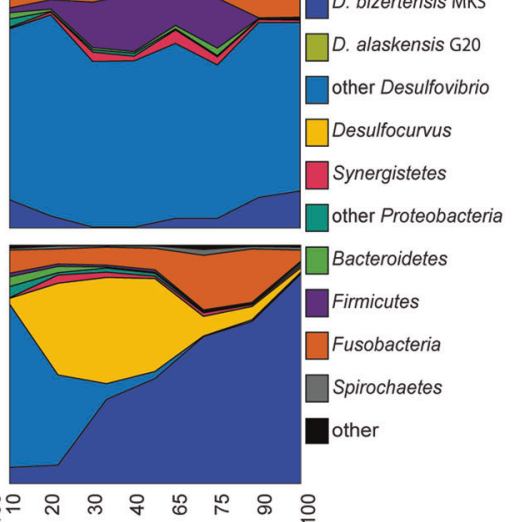
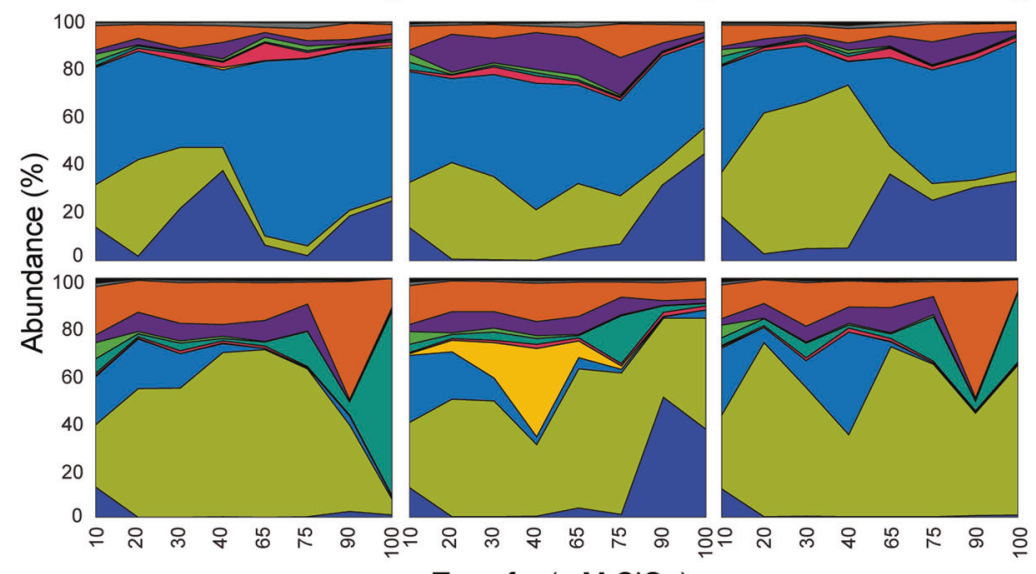

Transfer $\left(\mathrm{mM} \mathrm{ClO}_{4}^{-}\right)$

Changes to community structure could be explained by variability in the innate resistance to perchlorate of different SRM. We compared the resistance of MKS and a previously isolated Desulfovibrio $\mathrm{sp}$. from this community ( $D$. sp. BMSR) [18], both isolated prior to any adaptation on perchlorate (Fig. 2, Fig. S2). Although the two strains did not differ in their IC50 values, MKS had a significantly less steep hillslope ( $p=0.0044$, Extra sum-of-squares $F$ test), and was therefore less inhibited at higher concentrations of perchlorate $(>20 \mathrm{mM})$, growing slowly in even $50-100 \mathrm{mM}$ (Fig. S2). G20 was more resistant than BMSR ( $p=0.04$, Extra sum-of-squares $F$ test) and grew faster at $<50 \mathrm{mM}$ perchlorate than both other Desulfovibrio sp. tested (Fig. 2, Fig. S2). The Desulfocurvus spp. from this community was 
Fig. 1 a Experimental design: serial transfers of three native sulfatereducing communities (top panel) and three G20-augmented communities (bottom panel) in increasing concentrations of perchlorate, up to $100 \mathrm{mM}$. DNA was sampled at each transfer and used for 16s rRNA amplicon sequencing. Post adaptation, 8 isolates of MKS and 13 isolates of G20 were obtained from adapted communities for wholegenome sequencing. b IC50 values for control (patterned) and adapting (solid) native communities (top panel) and G20-augmented communities (bottom panel). Bars represent $95 \%$ confidence interval. For native communities, all dose-response curves generate significantly different IC50 values, except control 1 vs control 2 ( $p<$ 0.0001 , extra sum-of-squares $F$ test, Graphpad Prism). For G20augmented communities, all IC50s differ significantly from one another $(p<0.0001)$, except control 1 vs. control $2 / 3$ and control 2 vs. control 3. c Community profiles from $16 \mathrm{~S}$ rRNA amplicon sequencing for the independently transferred control and adapting replicate communities, over the course of successive transfers in increasing perchlorate concentrations

not isolated but was previously shown to persist in chemostats treated with $50 \mathrm{mM}$ perchlorate [18]. These results suggested that initial adaptation of communities to perchlorate was through an ecological mechanism: the elimination of less resistant taxa. We next explored beneficial adaptations acquired by the ecologically successful SRM (MKS and G20), allowing them to grow robustly in $100 \mathrm{mM}$ perchlorate.

\section{Mutation of the sulfate adenylyltransferase (sat) was not a dominant mechanism of adaptation to perchlorate}

The most abundant strains in each sulfate-reducing community (MKS and G20) were isolated and whole-genome sequenced to identify genetic mechanisms of adaptation. Because genomes might contain both adaptive and nonadaptive mutations, sequences from adapted and nonadapted conditions were compared to determine mutations specific to perchlorate adaptation. We hypothesized that adaptation would target the sulfate adenylyltransferase (sat), as had been observed previously in pure culture experiments with $D$. alaskensis G20 [17].

Single-colony MKS isolates from adapted communities (eight total) were whole-genome sequenced and compared compared with total community sequencing reads from controls (strain MKS could not be isolated from control communities) and the reference genome. Of the small set of mutations that occurred in adapted but not control MKS (Table S2), no mutations were detected in sat.

In $D$. alaskensis $\mathrm{G} 20$, we initially used previously published methods to determine the frequency of the sat $(709 \mathrm{~T}$ $>$ G) variant that had arisen repeatedly during previous adaptation experiments [17]. This analysis revealed that only in the first community did $\operatorname{sat}(709 \mathrm{~T}>\mathrm{G})$ arise, and as of the $100 \mathrm{mM}$ transfer, it constituted only $0.5 \%$ of all sat

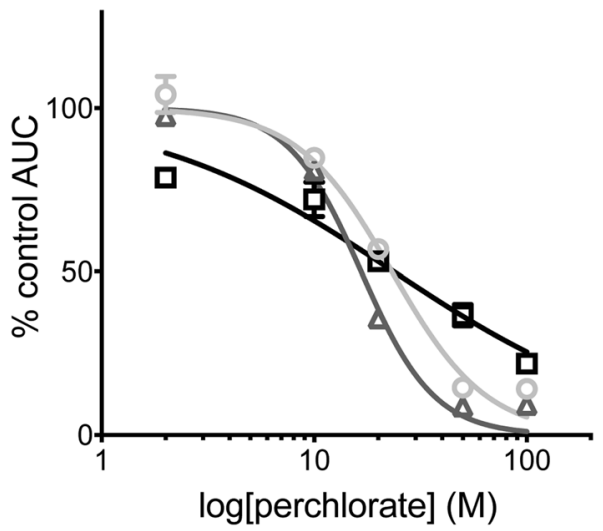

Fig. 2 Dose-response curves for MKS (black squares), D. sp. BMSR (gray triangles) and G20 (light gray circles). Error bars represent SD from four replicates. AUC $=$ area under the curve. IC50 values do not differ significantly between $D$. sp. BMSR and MKS ( $p=0.1179$, Extra sum-of-squares $F$ test); hillslope values do ( $p=0.0044$, Extra sum-ofsquares $F$ test). IC50 of G20 vs. BMSR is significantly different at $p=$ 0.04 (Extra sum-of-squares $F$ test)

amplicons. Isolates of G20 from control and recovered adapted communities were also whole-genome sequenced and compared. Only three of thirteen isolates, all from community one, contained the mutation in sat (Table S3). All other G20 isolates contained a mutation in either Dde_0320 or Dde_0321, predicted signaling components.

The predominance of mutations in the signaling components over the $\operatorname{sat}(709 \mathrm{~T}>\mathrm{G})$ mutation previously observed might be explained by medium composition or by community effects. A comparison of the isolated $D$. alaskensis strain $\operatorname{Pg} 11$ containing the $\operatorname{sat}(709 \mathrm{~T}>\mathrm{G})$ mutation to strain Pg33, with an inactivation of the Dde_0320 gene, revealed that $\operatorname{Pg} 11$ was less competitive in the medium used in this study (APM) than that used in previous studies (MOLS4) [17] (Fig. S3AB). Two additional representative strains (Pat3 and Dde_0320::TN5, Table 1) showed the same pattern (Fig. S4). Direct competition of the SRM strains $\mathrm{Pg} 11$ and $\mathrm{Pg} 33$ in $100 \mathrm{mM}$ perchlorate-amended APM revealed that $\mathrm{Pg} 33$ outcompeted Pg11 and its domination was stable (Fig. S3C). In co-culture with fermentative organisms, Pg33 took longer to dominate, and did so less stably. Thus, it seems that both community and medium effects shift the cost/benefit of adaptive mechanisms. Regardless of the cause, in our community adaptations neither of the dominant SRM adapted primarily via mutations in the sat.

\section{Adaptation by decreasing perchlorate import}

The most common mutations in D. bizertensis MKS occurred in a locus containing a transcription factor, a transporter, and accessory genes (Fig. 3a). Six of eight isolates had mutations in the DNA-binding helix-turn-helix (HTH) motif of a cAMP receptor protein-like transcription 
A.

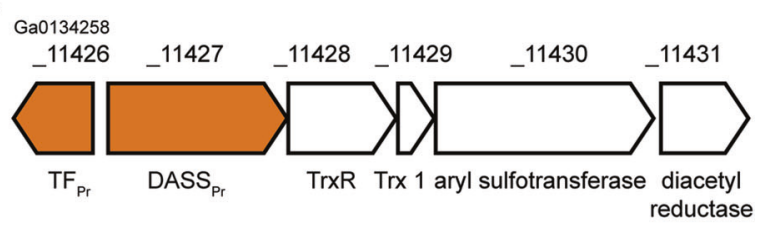

B.



C.

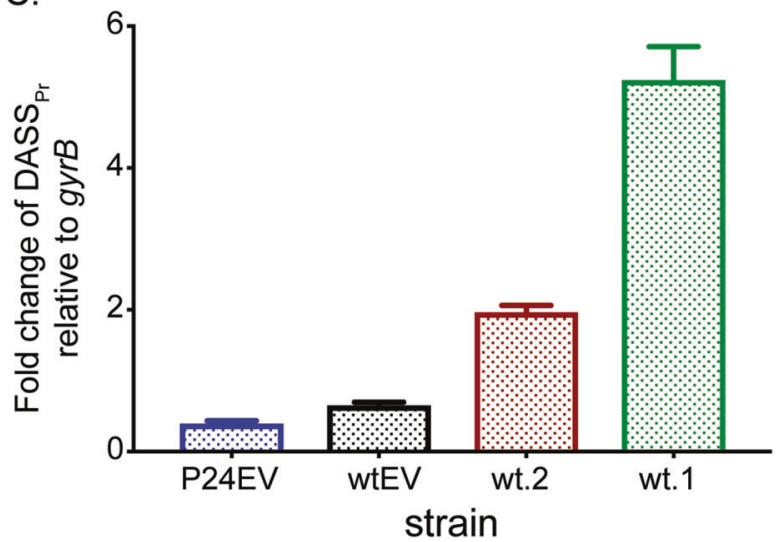

Fig. 3 a Genomic context of $\mathrm{TF}_{\mathrm{Pr}}$. Orange color indicates genes that are mutated in adapted strains. $\mathrm{TF}_{\mathrm{Pr}}=$ transcriptional regulator, Ga0134258_11426; DASS $\mathrm{Pr}_{\mathrm{Pr}}=$ divalent-anion:sodium symporter, Ga0134258_11427; TrxR = thioredoxin reductase; $\operatorname{Trx} 1=$ thioredoxin 1. b Growth of overexpression strains with (open symbols) and without (closed symbols) $100 \mathrm{mM}$ perchlorate. P24EV = adapted MKS strain P24 empty vector control; wt.1 = wild-type MKS overexpressing wt $\mathrm{TF}_{\mathrm{Pr}}$; wt. 2 = wild-type MKS overexpressing mutant (L209*) $\mathrm{TF}_{\text {Pr }}$; wtEV = wild-type MKS empty vector control. Values are average of three biological replicates; SD bars smaller than symbols. c Fold change in expression of DASS $\mathrm{Pr}_{\mathrm{Pr}}$ relative to $\operatorname{gyr} B$ across overexpression strains. Error bars represent $\mathrm{SD}$ of three biological replicates

factor $\left(\mathrm{TF}_{\mathrm{Pr}}, \mathrm{Ga} 0134258 \_11426\right)$, and all other isolates contained a nonsense mutation in the divalent anion:sodium symporter (DASS) family transporter (DASS $_{\mathrm{Pr}}$, Ga0134258_11427) gene directly upstream of $\mathrm{TF}_{\mathrm{Pr}}$. A mutation in $\mathrm{TF}_{\mathrm{Pr}}$ was also detected in the only sequenced MKS strain from the G20-augmented community two (the augmented community where MKS persisted) (Table S2).
To determine if mutations in this operon occur in pure culture adaptations, the ancestral MKS strain was independently adapted to $100 \mathrm{mM}$ perchlorate. All five adapted populations showed increased resistance to perchlorate compared with the two control populations (Fig. S5). Five single-colony adapted isolates across the populations were sequenced and all revealed mutations in either $\mathrm{TF}_{\mathrm{Pr}}$ or DASS $_{\text {Pr }}$ (Table S2). Therefore, in all biological conditions tested (pure culture, native community, G20-augmented community), mutations in $\mathrm{TF}_{\mathrm{Pr}}$ or DASS $\mathrm{Pr}_{\mathrm{Pr}}$ were correlated with perchlorate resistance in MKS.

Comparative genomics suggested that $\mathrm{TF}_{\mathrm{Pr}}$ regulates expression of the downstream operon. The operon is conserved across four other SRM: Desulfovibrio bizertensis DSM 18034, Desulfofustis glycolicus DSM 9705, Mailhella massiliensis Marseille-P3199, and Desulfobulbus sp. ORNL (Fig. S6, Table S4). In three of these organisms (MKS, DSM 9705 and Marseille-P3199) there is an inverted repeat upstream of the operon, which may serve as a TF binding site. Each respective genome was scanned for the presence of additional respective TF binding sites, and none were found. Thus, $\mathrm{TF}_{\mathrm{Pr}}$ likely regulates expression only of the downstream operon.

We hypothesized that increased resistance to perchlorate was caused by inactivation of the DNA-binding ability of $\mathrm{TF}_{\mathrm{Pr}}$, thereby changing expression of the downstream operon. In agreement with this, one of the adapted strains containing a mutation in the HTH domain of $\mathrm{TF}_{\mathrm{Pr}}$, strain P24 (Table 1), had significantly lower expression of $\mathrm{DASS}_{\mathrm{Pr}}$ as compared with wild-type MKS $(p<0.0001$, Mann-Whitney test) (Fig. S7). Because decreasing transcription initiation by the $\mathrm{TF}_{\mathrm{Pr}}$ correlated with increased fitness in $100 \mathrm{mM}$ perchlorate, we tested whether increasing $\mathrm{TF}_{\mathrm{Pr}}$ expression harmed the fitness of MKS in these conditions. The following strains were constructed (Table 1): overexpression of wild-type $\mathrm{TF}_{\mathrm{Pr}}$ (wt.1), overexpression of P24 mutant $\mathrm{TF}_{\mathrm{Pr}}$ (wt.2), and an empty vector control (wtEV) in the wild-type MKS background, and also an empty vector control in the P24 background (P24EV). Under our growth conditions, without perchlorate, all strains grew similarly (Fig. 3b). With $100 \mathrm{mM}$ perchlorate, the greatest growth occurred in the P24 control (P24EV), followed by the wild-type control (wtEV) and the overexpression of the mutant $\mathrm{TF}_{\mathrm{Pr}}$ (wt.2); the strain overexpressing the wild-type $\mathrm{TF}_{\mathrm{Pr}}$ did not grow. RT-qPCR indicated that expression of DASS $\mathrm{Pr}_{\mathrm{Pr}}$ matched the gradient of "intact" $\mathrm{TF}_{\mathrm{Pr}}$ levels in each strain: from lowest to highest, P24EV, wtEV, wt.2, and wt.1 (Fig. 3c). The degree of resistance to perchlorate was strongly inversely correlated with expression of the DASS $_{\text {Pr }}$ transporter.

A simple explanation for the inverse correlation between DASS $S_{\mathrm{Pr}}$ expression and perchlorate resistance is 

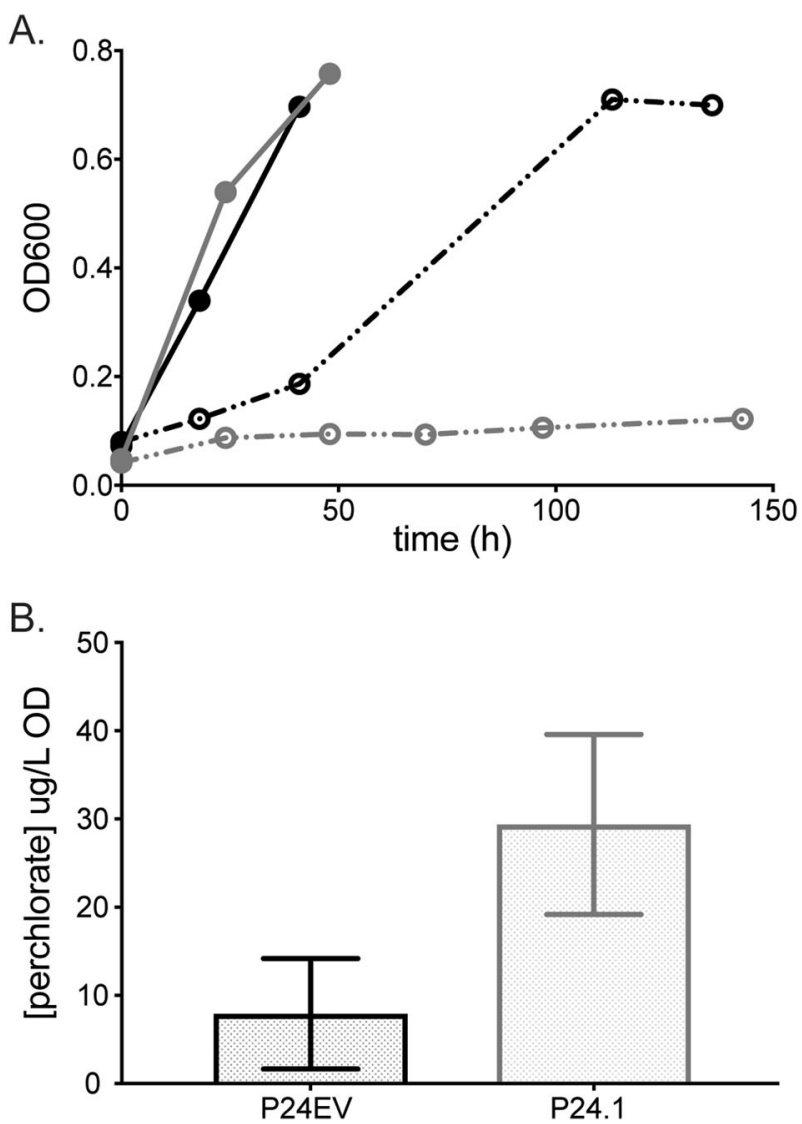

Fig. 4 a Growth of strains P24EV (black, adapted MKS strain P24 empty vector control) and P24.1 (gray, adapted MKS strain P24 overexpressing $\mathrm{DASS}_{\mathrm{Pr}}$ ) with (open symbols) and without (closed symbols) $100 \mathrm{mM}$ perchlorate. Values are average of three biological replicates; SD bars smaller than symbols. b Average normalized intracellular perchlorate concentration of P24EV and P24.1. Error bars are SD of three biological replicates

that $\mathrm{DASS}_{\mathrm{Pr}}$ is permeable to perchlorate. If true, decreasing expression of the transporter would decrease perchlorate import into cells and thus reduce competitive inhibition of Sat. Direct overexpression of $\mathrm{DASS}_{\mathrm{Pr}}$ in adapted strain P24 (P24.1) rendered the strain sensitive to perchlorate compared with the empty vector control (Fig. 4a). When incubated in the presence of $100 \mathrm{mM}$ perchlorate, P24.1 showed significantly more intracellular perchlorate than $\mathrm{P} 24 \mathrm{EV}(p=0.046$, Unpaired $t$ test $)$ (Fig. 4b). In addition, overexpression of the transporter in the wild-type MKS background also increased sensitivity of that strain (data not shown). These data indicate that DASS $_{\text {Pr }}$ is detrimental to fitness under high concentrations of perchlorate because it transports perchlorate into cells. In summary, strain MKS adapted to perchlorate by inactivating this transporter, via a nonsense mutation directly in the transporter or by mutating $\mathrm{TF}_{\mathrm{Pr}}$, responsible for its expression, thereby decreasing perchlorate import into cells (Fig. 7a).
Adaptation by increasing sulfate import

All sequenced perchlorate-adapted $D$. alaskensis G20 isolates that did not contain mutations in the sat, contained a mutation in the signaling proteins Dde_0320 (seven isolates) or Dde_0321 (three isolates) (Table S3). Dde_0320 encodes a histidine kinase with a cytoplasmic kinase domain and an extracellular sensory domain. Dde_0321 encodes a protein with a predicted response regulator receiver domain. The resistance phenotype for the histidine kinase null mutation was confirmed by comparing the growth of wild-type G20, adapted strain Pg33 (Table 1) and strain Dde_0320:TN5 (Table 1, courtesy of Adam Arkin). Disruption of the histidine kinase was sufficient for growth with $100 \mathrm{mM}$ perchlorate (Fig. 5b). We predicted that the genes regulated by the histidine kinase were responsible for adaptation to perchlorate.

The Dde_0320 histidine kinase is part of an operon, which includes genes encoding $\sigma^{54}$-dependent Fis family transcriptional regulator Dde_0318, hypothetical protein Dde_0319, protein Dde_0321 with a response-regulator receiver domain, and histidine kinase-like protein Dde_0322 (Fig. 5a). The operon has a predicted $\sigma^{54}$ dependent promoter, as does the downstream Dde_0323Dde_0328 operon, which contains genes encoding DASS family transporter Dde_0326, CBS domain protein Dde_0325, and four proteins putatively involved in signal transduction [40]. Dde_0319, Dde_0320, and Dde_0321 have been previously identified as detrimental under perchlorate and fluorophosphate stress (another specific inhibitor of SRM) [9, 41]. Thus, as this locus is consistently associated with perchlorate stress, we term genes Dde_0318-Dde_0328 the "perchlorate cluster" and henceforth refer to the transcriptional regulator (Dde_0318) as PclR; the histidine kinase (Dde_0320) as PclS; and the transporter (Dde_0326) as PclT.

Comparative genomics suggested that PclR controls expression of both its own and the downstream operon. Orthologs of PclS were identified in Desulfovibrio vulgaris Hildenborough (DvH) and Desulfovibrio vulgaris Miyazaki (DvM) (Fig. 5a) [40, 42]; in DvM, as in G20, the ortholog was detrimental to fitness under perchlorate stress [43]. Both DvH and DvM operons are regulated by Fis family $\sigma^{54}$-dependent transcriptional regulators DVU2956 in DvH and DvMF_1763 in DvM [42]. G20 lacks an ortholog to DVU2956/DvMF_1763 and the entire locus contains only one gene encoding a regulatory DNA-binding protein, $p c l R$ (Fig. 5a). Conserved binding sites for PclR were identified upstream of both operons in the perchlorate cluster (Supplemental Methods and Table S5.1). A whole-genome scan revealed three additional putative binding sites: upstream of Dde_0191, Dde_0218, and Dde_0683/Dde_0685, encoding a kinase, a methyl-accepting chemotaxis sensory transducer, and a cytochrome c family protein/ formylmethanofuran 
A.

Desulfovibrio alaskensis

$\mathrm{G} 20$

Cosufovibio vulgaris

Desulfovibrio vulgaris
Hildenborough
Desulfovibrio vulgaris

Desulfovibrio vulgaris
Miyazaki

Desulfomicrobium baculatum

DSM 4028
Desulfotalea psychrophila

LSv54

DCB-1, DSM 6799

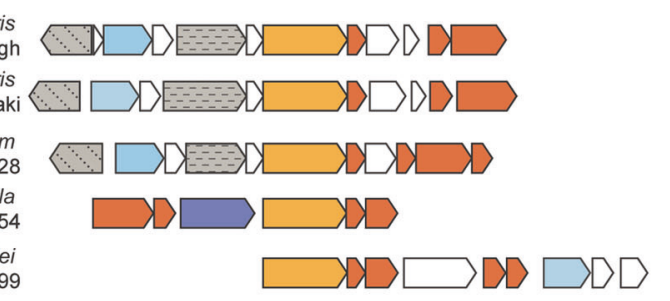

Dde_0318 $0319 \quad 0320 \quad 0321 \quad 03220323032403250326 \quad 0327 \quad 0328$

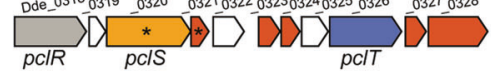

$\square X \square \square D D \square D \square$
B.

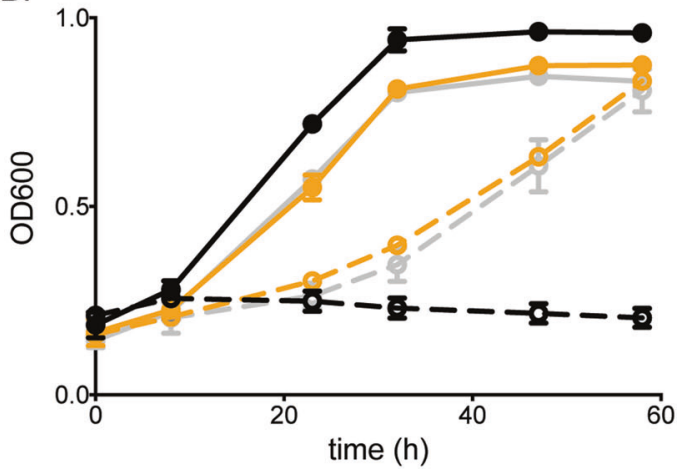

Fig. 5 a The "perchlorate cluster" and homologous operons in closely related SRM. $p c l R=\sigma^{54}$-dependent Fis family transcriptional regulator (Dde_0318); pclS = histidine kinase (Dde_0320); pclT = divalent-anion:sodium symporter (DASS) family transporter (Dde_0326); gray $=\sigma^{54}$-dependent Fis family transcriptional regulator, similarly patterned are orthologous; yellow = histidine kinase; orange $=$ protein with response regulator receiver domain; dark blue $=$ DASS family transporters; light blue $=$ TSUP family transporter. Asterisk

dehydrogenase subunit, respectively (Table S5.1). From this analysis, we conclude that PclR is the regulatory protein necessary for activation of both $\sigma^{54}$-dependent promoters in the perchlorate cluster, and, possibly of some other genes.

In order to determine how PclS fits into this regulatory network, we compared transcriptional profiles of wild-type G20 vs. the Dde_0320::TN5 strain, both in the absence (no stress) and presence of $100 \mathrm{mM}$ perchlorate (stress), using RNAseq. In both stress and no stress conditions, the region upstream of PclR had significantly greater expression (213x and 300× respectively) in Dde_0320::TN5 compared with wild type (Table S5.2 and S5.3). The downstream genes of the perchlorate cluster (Dde_0318-Dde_0319 and Dde_0323-Dde_0326) were also overexpressed ( $\sim$ two to four-fold). From the computationally identified targets of PclR described above, only Dde_0218 was differentially expressed both under stress and no stress conditions $(\sim 9 \times$ up-regulated in Dde_0320::TN5) (Table S5.4). Additional sets of genes with differential expression (Table S5.5) are not discussed here (see: Discussion). We propose that PclS senses a signal and directly or via a regulatory cascade modulates the activity of PclR, thereby repressing transcription of the perchlorate cluster. Inactivation of PclS, hence, increases transcription of all gene targets (Fig. 7b).

The regulon of PclR contains only one gene with putative activity towards sulfate or sulfate analogs: PclT, another DASS family transporter. The closest studied homolog of PclT, HRM2_40290 from Desulfobacterium autotrophicum HRM2, is a putative sulfate symporter expressed under high sulfate conditions and co-localized with regulatory elements [44]. We hypothesized that PclT is also a tightly regulated sulfate symporter. Direct measurements of intracellular sulfate and perchlorate in strain indicates genes which sustained mutations during adaptation to perchlorate. Operons (in order, top to bottom): Dde_0318-Dde_0328, DVU2956-DVU2967, DvMF_1763-DvMF_1773, Dbac_2263Dbac_2272, DP1487-DP1492, and Desti_4439-Desti_4447. b Growth in MOLS4 medium of wild- type G20 (black), the Dde_0320:: TN5 strain (yellow) and the Pg33 adapted strain (gray) with (open symbols) and without (closed symbols) $100 \mathrm{mM}$ perchlorate. Bars represent SD of three replicates. For description of strains, see Table 1

Dde_0320::TN5 vs. wild type found that although intracellular perchlorate did not differ $(p=0.32)$, sulfate was above detection in the Dde_0320::TN5 strain and below detection in wild type (Table S6) suggesting a highly specific transport function. Even with a conservative estimate of sulfate in wild type, placing it at the detection limit, this difference is significant $(p=0.004)$. To confirm sulfate transport by PclT, we heterologously expressed either PclT alone, or the entire operon (Dde_0323-Dde_0328) in E. coli strain JW2415 (deficient in sulfate uptake $(\Delta$ cysA)) (Table 1) [38]. Both constructs complemented the sulfate uptake deficiency phenotype (Fig. 6). Thus, PclT can transport sulfate; other genes in its operon may modulate this transport activity. In summary, G20 adapted to perchlorate by inactivating PclS, thus increasing transcription of the perchlorate cluster and specifically sulfate import via PclT. Increased intracellular sulfate, relative to perchlorate, can overcome the competitive effects of perchlorate on Satmediated sulfate turnover (Fig. 7b).

\section{Discussion}

The experiments outlined above describe community-wide and species-specific responses to increased perchlorate concentrations. Both native and G20-augmented communities initially adapted to the inhibitor via changes in community structure. Using a combination of isolation, whole genome sequencing, and genetics we show that across the two dominant adapting SRM, adaptation to perchlorate targets regulatory elements controlling anion transport systems, thereby modulating the intracellular concentrations of sulfate and perchlorate. 
Fig. 6 Growth of strains jwEV (black, E. coli JW2415 empty vector control), jw.1 (light gray, E. coli JW2415 expressing pclT), and jw.2 (dark gray, $E$. coli JW2415 expressing the entire perchlorate cluster) in M63 minimal medium with sulfur sources: a $250 \mathrm{uM}$ sulfate or b $250 \mathrm{uM}$ cysteine
A.

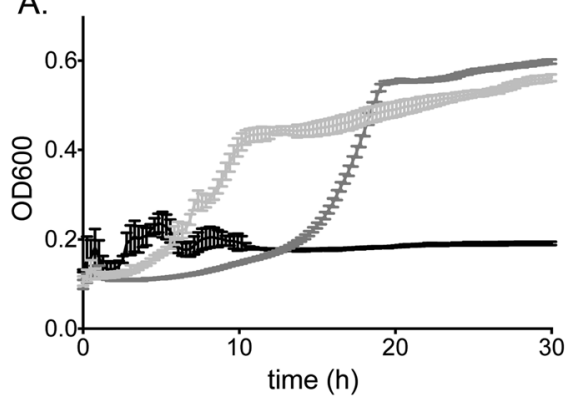

B.

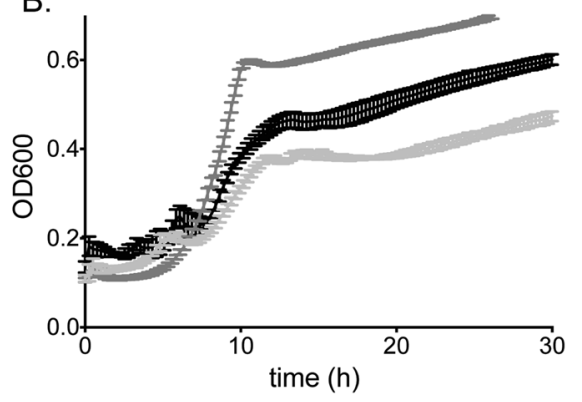

Selection initially acted on species abundances (ecological sorting), allowing for the expansion of innately more resistant SRM [45]. Initially dominant Desulfovibrio spp. made way to a Desulfocurvus sp., which in turn made way to MKS or G20. In our community, the sulfate-reducing niche was consistently around $85 \%$; thus, SRM had to compete for this niche during adaptation. G20 was only competitive in perchlorate-adapting conditions. In control communities G20 was outcompeted, but strikingly, caused an increase in MKS; this is indicative of a possible synergy between the two, or else G20 negatively affected other Desulfovibrio which otherwise outcompete MKS. In contrast to the consistency observed across replicate native adapting communities, the added competition from G20 created variability in augmented communities. G20 has a slight advantage at low perchlorate concentrations, and eliminated much of its competition early on in the adaptation. However, this early advantage was tightly balanced by slower growth in the APM medium and at higher perchlorate concentrations. Stochasticity may have played a larger role under these conditions: any minimally-present populations with a slight advantage at $>40 \mathrm{mM}$ perchlorate would have had a chance for rampant outgrowth.

Genomic adaptation ultimately allowed for robust sulfate reduction at $100 \mathrm{mM}$ perchlorate. In Desulfovibrio bizertensis MKS, adaptation decreased the transcription initiation ability of $\mathrm{TF}_{\mathrm{Pr}}$, which decreased expression of the DASS $\mathrm{Pr}_{\mathrm{Pr}}$ transporter and consequently, non-specific perchlorate transport into cells. In Desulfovibrio alaskensis G20, adaptation targeted PclS, a histidine kinase, leading to increased transcription of the perchlorate cluster, including PclT, a sulfate symporter. Inactivation of PclS led to increased intracellular sulfate, via PclT. Both decreased perchlorate transport and increased sulfate transport increased the intracellular sulfate:perchlorate ratio, thus overcoming the effects of competitive inhibition (Fig. 7).

Previous work suggested that SRM adapt to perchlorate by mutating the conserved sat, responsible for catalyzing the first step of sulfate reduction [17]. In contrast, this study finds that SRM in an ecological context adapt to perchlorate via mutations in transport systems or the regulatory systems controlling them: targets that are less conserved and much more redundant. Model SRM DvH, for example, has 72 response regulators, the majority of which lack a DNAbinding domain and instead likely act via protein-protein interactions [46, 47]. The diversity of regulatory systems in SRM reflect how these organisms cope with stress [46]; unsurprisingly, they represent an easy target for adaptation.

Sulfate transport in SRM is similarly complex and redundant: G20 has 11 putative sulfate transporters, whose exact function and relative importance remain uncharacterized [48]. Transport systems have been previously defined as targets of various stressors: in G20, DvH, and DvM, nitrate stress targets genes of the "nitrate cluster", which include a CysZ-type permease (Dde_0598) $[9,43,49]$. Nitrate resistance is attributed to the loss of this permease and decreased nitrate entry into cells. Similarly, the TunR2 regulator in DvH and other SRM was predicted to protect against inhibition by molybdate/tungstate by putatively increasing sulfate uptake via TSUP and SulP family transporters [50].

SRM genomes contain on average three DASS family transporters [48]. Few transporters of this family have been experimentally characterized. An exception is the PerO anion permease of Rhodobacter capsulatus, which when inactivated increases resistance to molybdate, tungstate, and vanadate [51]. Moreover, sulfate transport and increased resistance were confirmed for five homologous permeases across the Proteobacteria [52]. PerO is a general, lowaffinity anion transporter-i.e. promiscuous-setting a precedent for the non-specific transport observed by DASS $_{\mathrm{Pr}}$. In Desulfobacterium autotrophicum, DASS family transporters are either expressed constitutively, or are tightly regulated [44]. PclT is homologous to HRM2_40290 from D. autotrophicum, which is expressed only in "high" sulfate $(\sim 15 \mathrm{mM})$. Collectively, our study and others cited above define sulfate/anion transport as a critical, highly redundant and poorly understood function in SRM.

The native function of the targeted operon in MKS remains unknown. Operons regulated by CRP-like transcription factors and encoding transporters, and thioredoxin/thioredoxin reductases are implicated in nitrate and oxidative stress and in $\mathrm{U}(\mathrm{IV}) / \mathrm{Cr}(\mathrm{VI})$ reduction [53-55]. It is possible that in MKS this operon is involved in cytoplasmic redox balance. The 


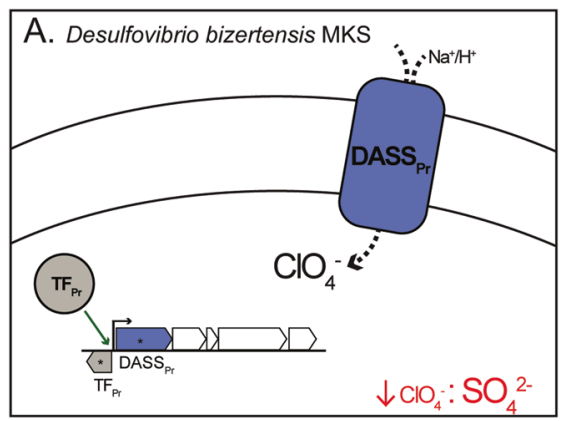

Fig. 7 Adaptation to perchlorate targets anion transport in both Desulfovibrio bizertensis MKS and Desulfovibrio alaskensis G20, increasing intracellular sulfate:perchlorate ratios and alleviating effects of competitive inhibition. a In MKS, $\mathrm{TF}_{\mathrm{Pr}}$ regulates expression of the downstream DASS $_{\mathrm{Pr}}$, which is leaky towards perchlorate. Inactivation of the transporter or TF leads to decreased perchlorate entry into cells. b In G20, PclS represses the activity of PclR (directly or via a regulatory cascade);

native function of the perchlorate cluster may be related to sulfate uptake via PclT. Recently, the homologous operon in DvH was linked to biofilm formation via another $\sigma^{54}$-dependent regulator, DVU2956, which is absent in G20 [56]. Results from our study do not support an obvious role related to biofilm formation, and comparison to the described targets of DVU2956 yields minimum overlap [56]. RNAseq data, however, identified several additional loci/regulons significantly up-regulated in the Dde_0320::TN5 strain (Table S5.5). The first consisted of genes involved in fumarate reduction, and critical for growth on malate or fumarate, and by pyruvate fermentation (Dde_1250-Dde_1258) [32, 33, 57]. G20 grown in pyruvate/sulfate medium was previously shown to display increased resistance against perchlorate, perhaps as a result of partial fermentation of pyruvate, consuming reducing equivalents which have built up by the perchloratemediated inhibition of sulfate reduction $[9,18]$. It is possible that the up-regulation of fumarate reduction genes (and the downregulation of the pyruvate ferredoxin oxidoreductase) allow for a similar process in the Dde_0320::TN5 strain.

The ZraR and HcpR regulons are also up-regulated in the Dde_0320::TN5 strain. In DvM and DvH, ZraR is important for chlorate and oxidative stress [58]; in G20, this regulon is induced upon exposure to molybdenum, suggesting its involvement in molybdenum tolerance [59]. The HcpR regulon consists of genes putatively involved in energy production/conversion. Across G20, DvH, DvM, and Desulfovibrio gigas, these genes are important under nitrosative and perchlorate stress $[53,60,61]$. Whether or not there is true cross-regulation between PclS and the transcriptional regulators controlling the above regulons remains to be addressed; however, cross or branched regulation are not uncommon [42, 53, 62].

Recent studies have highlighted the importance of a community context or "natural" environment in determining

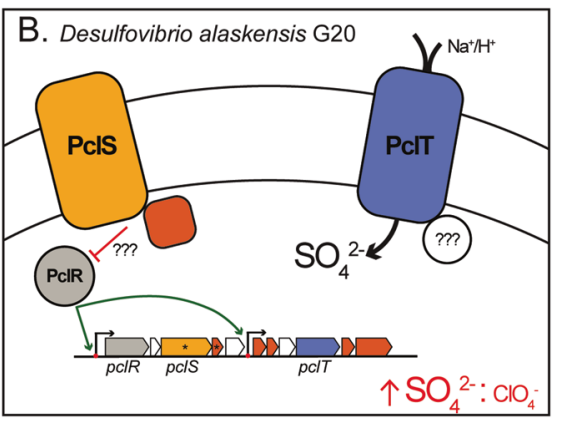

PclR activates transcription of the perchlorate cluster, including sulfate transporter, PclT. Inactivation of $p c l S$ thus causes increased sulfate entry into cells. Other genes in the cluster, or elsewhere in the genome may modulate the transport activity of PclT. Asterisk indicates genes in which mutations were sustained over the course of adaptation to perchlorate; red dot $=\sigma^{54}$-depdendent promoters

evolutionary outcomes, via mechanisms such as increasing costs of adaptive mutations or decreasing niche availabilities $[26,27]$. Previous work in G20 has shown that the $\operatorname{sat}(709 \mathrm{~T}>$ G) mutation arises across all independently adapted populations [17]. Here, we saw this mutation arise only in one community, and this community showed the lowest level of sulfidogenesis in $100 \mathrm{mM}$ perchlorate and a concomitant crash of the G20 population. We have shown that in the APM medium used for community adaptations, strains with an inactivation of PclS have increased relative fitness compared to strains with sat (709T $>$ G). It is possible that the community context changes the adaptive landscape in additional ways: supplying different donors, altering the $\mathrm{pH}$, or modulating population sizes [24]. Indeed, G20, MKS, and other SRM competed for the sulfatereducing niche throughout the adaptation. This tight competition may have limited the rise of more costly mutations, and may explain why we failed to see adaptation of sulfidogenic communities in previous studies [18]. Finally, the specificity and regulation of two DASS family transporters examined herein emphasize the importance of anion transport as a conserved mechanism used by SRM in communities, when faced with sulfate analog stress. Future work will need to address the paucity of information on this subject for a predictive understanding of long-term SRM inhibition in natural environments.

Acknowledgements We would like to thank Andrew Jackson (Texas Tech University) for intracellular sulfate and perchlorate analysis, Grant Zane and the Judy Wall lab (University of Missouri) for discussions regarding overexpression in SRM, Angeliki Marietou (Aarhus University, Denmark) for discussions regarding SRM transporters, Misha Mehta for the FREQseq experiment, Tyler P. Barnum for editing the manuscript and figures, and Anchal Mehra and Nicholas Garcia for general support. Funding support for research on microbial sulfate reduction was generously provided by the Energy Biosciences Institute through their BP research program to JDC. All sequencing data is publicly available under the NCBI BioProject ID PRJNA560652. 


\section{Compliance with ethical standards}

Conflict of interest The authors declare that they have no conflict of interest.

Publisher's note Springer Nature remains neutral with regard to jurisdictional claims in published maps and institutional affiliations.

\section{References}

1. Oremland RS, Capone DG. Use of "Specific " inhibitors in biogeochemistry and microbial ecology. Adv Microb Ecol. 1988;10:285-383.

2. Carlson HK, Mullan MR, Mosqueda LA, Chen S, Arkin MR, Coates JD. High-throughput screening to identify potent and specific inhibitors of microbial sulfate reduction. Environ Sci Technol. 2017;51:7278-85.

3. Zhu W, Winter MG, Byndloss MX, Spiga L, Duerkop BA, Hughes ER, et al. Precision editing of the gut microbiota ameliorates colitis. Nature. 2018;553:208-11.

4. Muyzer G, Stams AJM. The ecology and biotechnology of sulphate-reducing bacteria. Nat Rev Microbiol. 2008;6:441-54.

5. Enning D, Garrelfs J. Corrosion of iron by sulfate-reducing bacteria: New views of an old problem. Appl Environ Microbiol. 2014;80:1226-36.

6. Postgate J. Competitive inhibition of sulfate reduction by selenate. Nature. 1949;164:670-1.

7. Postgate BYJR. Competitive and non-competitive inhibitors of bacterial sulphate reduction. J Gen Micriobiol. 1952;6:128-42.

8. Gieg LM, Jack TR, Foght JM. Biological souring and mitigation in oil reservoirs. Appl Microbiol Biotechnol. 2011;92:263-82.

9. Carlson HKHK, Kuehl JVJV, Hazra ABAB, Justice NBNB, Stoeva MKMK, Sczesnak A, et al. Mechanisms of direct inhibition of the respiratory sulfate-reduction pathway by (per)chlorate and nitrate. ISME J. 2015;9:1295-305.

10. Carlson HKHK, Stoeva MKMK, Justice NBNB, Sczesnak A, Mullan MRMR, Mosqueda LALA, et al. Monofluorophosphate is a selective inhibitor of respiratory sulfate-reducing microorganisms. Environ Sci Technol. 2015;49:3727-36.

11. Engelbrektson A, Hubbard CG, Tom LM, Boussina A, Jin YT, Wong $\mathrm{H}$, et al. Inhibition of microbial sulfate reduction in a flowthrough column system by (per)chlorate treatment. Front Microbiol. 2014;5:315.

12. Cheng Y, Hubbard CG, Li L, Bouskill N, Molins S, Zheng L, et al. Reactive transport model of sulfur cycling as impacted by perchlorate and nitrate treatments. Environ Sci Technol. 2016;50:7010-8.

13. Hanna E, MacRae IJ, Medina DC, Fisher AJ, Segel IH. ATP sulfurylase from the hyperthermophilic chemolithotroph Aquifex aeolicus. Arch Biochem Biophys. 2002;406:275-88.

14. Hanna E, Ng KF, MacRae IJ, Bley CJ, Fisher AJ, Segel IH. Kinetic and Stability Properties of Penicillium chrysogenum ATP Sulfurylase missing the C-terminal regulatory domain. J Biol Chem. 2004;279:4415-24.

15. Kuehl JV, Price MN, Ray J, Wetmore KM, Esquivel Z, Kazakov $\mathrm{AE}$, et al. Functional genomics with a comprehensive library of transposon mutants for the sulfate-reducing bacterium Desulfovibrio alaskensis G20. MBio. 2014;5:1-13.

16. Christensen GA, Zane GM, Kazakov AE, Li X, Rodionov DA, Novichkov PS, et al. Rex (Encoded by DVU_0916) in Desulfovibrio vulgaris Hildenborough is a repressor of sulfate adenylyl transferase and is regulated by NADH. J Bacteriol. 2015;197:29-39.

17. Mehta-Kolte MG, Stoeva MK, Mehra A, Redford SA, Youngblut MD, Zane G, et al. Adaptation of Desulfovibrio alaskensis G20 to perchlorate, a specific inhibitor of sulfate reduction. Environ Microbiol. 2019;21:1395-1406.

18. Stoeva MK, Nalula G, Garcia N, Yiwei C, Engelbrektson A, Carlson $\mathrm{H}$, et al. Resistance and resilience of sulfidogenic communities in the face of the specific inhibitor perchlorate. Front Microbiol. 2019;10:654.

19. Elena SF, Lenski RE. Evolution experiments with microorganisms: the dynamics and genetic bases of adaptation. Nat Rev Genet. 2003;4:457-69.

20. Romero PA, Arnold FH. Exploring protein fitness landscapes by directed evolution. Nat Rev Mol Cell Biol. 2009;10:866-76.

21. Portnoy VA, Bezdan D, Zengler K. Adaptive laboratory evolution-harnessing the power of biology for metabolic engineering. Curr Opin Biotechnol. 2011;22:590-4.

22. Mozhayskiy V, Tagkopoulos I. Guided evolution of in silico microbial populations in complex environments accelerates evolutionary rates through a step-wise adaptation. BMC Bioinform. 2012;13:Suppl 10:S10.

23. Dragosits M, Mattanovich D. Adaptive laboratory evolution principles and applications for biotechnology. Micro Cell Fact. 2013;12:1-17.

24. Turcotte MM, Corrin MSC, Johnson MTJ. Adaptive evolution in ecological communities. PLoS Biol. 2012;10:e1001332.

25. Lawrence D, Fiegna F, Behrends V, Bundy JG, Phillimore AB, Bell T, et al. Species interactions alter evolutionary responses to a novel environment. PLoS Biol. 2012;10:e1001330.

26. Gómez P, Buckling A. Bacteria-phage antagonistic coevolution in soil. Science. 2011;332:106-9.

27. Gomez $P$, Buckling A. Real-time microbial adaptive diversification in soil. Ecol Lett. 2013;16:650-5.

28. Lenski RE. Experimental evolution and the dynamics of adaptation and genome evolution in microbial populations. ISME J. 2017;11:2181-94.

29. Engelbrektson AL, Cheng Y, Hubbard CG, Jin YT, Arora B, Tom LM, et al. Attenuating sulfidogenesis in a soured continuous flow column system with perchlorate treatment. Front Microbiol. 2018;9:1575.

30. Cline JD. Spectrophotometric determination of hydrogen sulfide in natural waters 1. Limnol Oceanogr. 1969;14:454-8.

31. Widdel F, Bak F. Gram-negative mesophilic sulfate-reducing bacteria. In The Prokaryotes 1992 (pp. 3352-78). Springer, New York, NY.

32. Keller KL, Rapp-Giles BJ, Semkiw ES, Porat I, Brown SD, Wall JD. New model for electron flow for sulfate reduction in Desulfovibrio alaskensis G20. Appl Environ Microbiol. 2014;80: 855-68.

33. Meyer B, Kuehl JV, Price MN, Ray J, Deutschbauer AM, Arkin $\mathrm{AP}$, et al. The energyconserving electron transfer system used by Desulfovibrio alaskensis strain G20 during pyruvate fermentation involves reduction of endogenously formed fumarate and cytoplasmic and membrane-bound complexes, Hdr-Flox and Rnf. Environ Microbiol. 2014;16:3463-86.

34. Picelli S, Björklund AK, Reinius B, Sagasser S, Winberg G, Sandberg R. Tn5 transposase and tagmentation procedures for massively scaled sequencing projects. Genome Res. 2014;24:2033-40.

35. Tjaden B. De novo assembly of bacterial transcriptomes from RNA-seq data. Genome Biol. 2015;16:1-10.

36. Keller KL, Wall JD, Chhabra S. Methods for engineering sulfate reducing bacteria of the genus Desulfovibrio. In: Methods in enzymology 2011 Jan 1 (Vol. 497, pp. 503-17). Academic Press.

37. Rahn-Lee L, Byrne ME, Zhang M, Le Sage D, Glenn DR, Milbourne $\mathrm{T}$, et al. A Genetic strategy for probing the functional diversity of magnetosome formation. PLoS Genet. 2015;11: e1004811. 
38. Baba T, Ara T, Hasegawa M, Takai Y, Okumura Y, Baba M, et al. Construction of Escherichia coli K-12 in-frame, single-gene knockout mutants: the Keio collection. Mol Syst Biol. 2006;2:2006.0008.

39. Jackson WA, Rao B, Sevanthi R, Estrada N, Anderson TA, et al. Global patterns and environmental controls of perchlorate and nitrate co-occurrence in arid and semi-arid environments. Geochim Cosmochim Acta. 2015;164:502-22.

40. Novichkov PS, Kazakov AE, Ravcheev DA, Leyn SA, Kovaleva GY, Sutormin RA, et al. RegPrecise 3.0 - A resource for genomescale exploration of transcriptional regulation in bacteria. BMC Genomics. 2013;14:1.

41. Dehal PS, Joachimiak MP, Price MN, Bates JT, Baumohl JK, Chivian D, et al. MicrobesOnline: an integrated portal for comparative and functional genomics. Nucleic Acids Res. 2010; 38:396-400.

42. Kazakov AE, Rajeev L, Chen A, Luning EG, Dubchak I, Mukhopadhyay A, et al. Sigma(54)-dependent regulome in Desulfovibrio vulgaris Hildenborough. BMC Genomics. 2015; $16: 1-16$.

43. Lo V, Shao W, Price M. Fitness browser. http://fit.genomics.lbl. gov/cgibin/myFrontPage.cgi. Accessed 9 Dec 2018.

44. Tarpgaard IH, Jørgensen BB, Kjeldsen KU, Røy H. The marine sulfate reducer Desulfobacterium autotrophicum HRM2 can switch between low and high apparent halfsaturation constants for dissimilatory sulfate reduction. FEMS Microbiol Ecol. 2017; 93:1-11.

45. De Mazancourt C, Johnson E, Barraclough TG. Biodiversity inhibits species' evolutionary responses to changing environments. Ecol Lett. 2008;11:380-8.

46. Zhou J, He Q, Hemme CL, Mukhopadhyay A, Hillesland K, Zhou A, et al. How sulphate-reducing microorganisms cope with stress: lessons from systems biology. Nat Rev Microbiol. 2011; 9:452-66.

47. Rajeev L, Luning EG, Altenburg S, Zane GM, Baidoo EEK, Catena $\mathrm{M}$, et al. Identification of a cyclic-di-GMP-modulating response regulator that impacts biofilm formation in a model sulfate reducing bacterium. Front Microbiol. 2014;5:1-13.

48. Marietou A, Røy H, Jørgensen BB, Kjeldsen KU. Sulfate transporters in dissimilatory sulfate reducing microorganisms: a comparative genomics analysis. Front Microbiol. 2018;9:1-21.

49. Korte HL, Fels SR, Christensen Ga, Price MN, Kuehl JV, Zane GM, et al. Genetic basis for nitrate resistance in Desulfovibrio strains. Front Microbiol. 2014;5:153.

50. Kazakov AE, Rajeev L, Luning EG, Zane GM, Siddartha K, Rodionov DA, et al. New family of tungstate-responsive transcriptional regulators in sulfate-reducing bacteria. J Bacteriol. 2013;195:4466-75.

51. Gisin J, Müller A, Pfänder Y, Leimkühler S, Narberhaus F, Masepohl BA. Rhodobacter capsulatus member of a universal permease family imports molybdate and other oxyanions. J Bacteriol. 2010;192:5943-52.

52. Hoffmann MC, Pfänder Y, Tintel M, Masepohl B. Bacterial PerO permeases transport sulfate and related oxyanions. J Bacteriol. 2017;199:pii: e00183-17.

53. Zhou A, Chen YI, Zane GM, He Z, Hemme CL, Joachimiak MP, et al. Functional characterization of Crp/Fnr-type global transcriptional regulators in Desulfovibrio vulgaris Hildenborough. Appl Environ Microbiol. 2012;78:1168-77.

54. Valette O, Tran TTT, Cavazza C, Caudeville E, Brasseur G, Dolla A, et al. Biochemical Function, molecular structure and evolution of an atypical thioredoxin reductase from Desulfovibrio vulgaris. Front Microbiol. 2017;8:1-16.

55. Li X, Krumholz LR. Thioredoxin is involved in $\mathrm{U}(\mathrm{VI})$ and $\mathrm{Cr}(\mathrm{VI})$ reduction in Desulfovibrio desulfuricans G20. J Bacteriol. 2009;191:4924-33.

56. Zhu L, Gong T, Wood TL, Yamasaki R, Wood TK. $\sigma_{54}$-Dependent regulator DVU2956 switches Desulfovibrio vulgaris from biofilm formation to planktonic growth and regulates hydrogen sulfide production. Environ Microbiol. 2019;1462-2920:14679.

57. Price MN, Ray J, Wetmore KM, Kuehl JV, Bauer S, Deutschbauer AM, et al. The genetic basis of energy conservation in the sulfate-reducing bacterium Desulfovibrio alaskensis G20. Front Microbiol. 2014;5:6-12.

58. Mukhopadhyay A, Redding AM, Joachimiak MP, Arkin AP, Borglin SE, Dehal PS, et al. Cell-wide responses to low-oxygen exposure in Desulfovibrio vulgaris Hildenborough. J Bacteriol. 2007;189:5996-6010.

59. Rivas MG, Carepo MSP, Mota CS, Korbas M, Durand MC, Lopes AT, et al. Molybdenum induces the expression of a protein containing a new heterometallic mo-fe cluster in Desulfovibrio alaskensis. Biochemistry. 2009;48:873-82.

60. He Q, He Z, Joyner DC, Joachimiak M, Price MN, Yang ZK, et al. Impact of elevated nitrate on sulfate-reducing bacteria: a comparative study of Desulfovibrio vulgaris. ISME J. 2010;4:1386-97.

61. da Silva SM, Amaral C, Neves SS, Santos C, Pimentel C, Rodrigues-Pousada C. An HcpR paralog of Desulfovibrio gigas provides protection against nitrosative stress. FEBS Open Bio. 2015;5:594-604.

62. Goulian M. Two-component signaling circuit structure and properties. Curr Opin Microbiol. 2010;13:184-9. 